\title{
Chronic Relapsing Inflammatory Optic Neuropathy
}

\author{
Ertuğrul Tan Yassa, ${ }^{1}$ (1) Berker Bakbak²
}

'Department of Ophthalmology, Asya Eye Hospital, Istanbul, Turkey

${ }^{2}$ Department of Ophthalmology, Selcuk University Faculty of Medicine, Konya, Turkey

\begin{abstract}
Chronic relapsing inflammatory optic neuropathy (CRION) is a form of recurrent, isolated, subacute optic neuropathy. A 33-year-old female presented at an outpatient clinic with a pain-ful reduction of vision in the left eye that had developed 10 days earlier. The patient provided a background history of 5 similar attacks over the past 5 years. CRION was diagnosed following ophthalmological and imaging examinations, which revealed optic neuritis without demye-lination. The patient was successfully treated with steroids. The early detection of CRION is important because of the associated risk of blindness if CRION is treated inappropriately. Keywords: Chronic relapsing inflammatory optic neuropathy; recurrent optic neuritis; steroid-dependent optic neuritis; steroid responsive.
\end{abstract}

Please cite this article as "Yassa ET, Bakbak B. Chronic Relapsing Inflammatory Optic Neuropathy. Med Bull Sisli Etfal Hosp 2019;53(4):437-440".

\begin{abstract}
A syndrome comprised of subacute visual loss, pain, and a clear and early response to systemic steroids is easily identifiable as inflammatory optic neuritis (ON). ON may occur as a manifes-tation of systemic autoimmune diseases, sarcoidosis, or central nervous system (CNS) demye-linating disease. An annual incidence of ON of 5.1 per 100.000 person-years and a prevalence rate of 115 per 100.000 has been reported. ${ }^{[1]}$ Although $\mathrm{ON}$ is frequently limited to a single episode, $3 \%$ to $5 \%$ of patients experience recurrent episodes (affecting either eye or both eyes sequentially or simultaneously), with a negative workup for multiple sclerosis (MS), neuromye-litis optica (NMO), or other potential causes. ${ }^{[2]}$ This condition is now called chronic relapsing inflammatory ON (CRION). In their original report on CRION, Kidd et al..$^{[3]}$ described pa-tients with bilateral inflammatory ON and recurrent relapses over time that worsened upon ster-oid or immunosuppression withdrawal. The detection of these individuals is important for pa-tient management targeted at the preservation of vision because there is a considerable risk of blindness. ${ }^{[4]}$
\end{abstract}

The present patient had experienced recurrent episodes of optic neuropathy over a period of 5 years with features that led to a diagnosis of CRION.

The patient provided written consent for publication of this case study.

\section{Case Report}

A 33-year-old female presented at the outpatient clinic with painful diminution of vision in the left eye developing over a period of 10 days. The pain persisted beyond the onset of visual loss. The patient provided a background history of 5 similar attacks over the previous 5 years involv-ing a single eye but never both simultaneously or sequential bilateral involvement. Each episode began with local pain, which increased with eye movement and visual loss progressed over the next 7 to 10 days. During these episodes, the patient's visual acuity varied between the percep-tion of light to finger counting at $1 \mathrm{~m}$. The interval between episodes varied between 2 and 6 months. Each episode remitted after the administration of intravenous steroids (methylpred-

Address for correspondence: Ertuğrul Tan Yassa, MD. Selcuklu Tip Fakultesi Alaaddin Keykubad Kampusu 42075, Konya, Turkey

Phone: +90 5335465731 E-mail: drberkerbakbak@yahoo.com

Submitted Date: November 14, 2017 Accepted Date: December 21, 2017 Available Online Date: November 20, 2019

${ }^{\circ}$ Copyright 2019 by The Medical Bulletin of Sisli Etfal Hospital - Available online at www.sislietfaltip.org

OPEN ACCESS This is an open access article under the CC BY-NC license (http://creativecommons.org/licenses/by-nc/4.0/) 
niso-lone, $1 \mathrm{~g}$ ) for 3 days and oral methylprednisolone at 1 $\mathrm{mg} / \mathrm{kg}$ for the following 11 days. Partial recovery of vision occurred upon remittance.

The patient did not have any temporal artery tenderness, and temporal pulses were palpable bi-laterally. There was no history suggestive of any other cranial nerve, motor, sensory, or auto-nomic system involvement. No relevant symptoms suggestive of connective tissue disease or autoimmune illness were noted historically. An examination revealed a relative afferent pupillary defect in the left eye. There was reduced visual acuity in the left eye, with finger counting at $1 \mathrm{~m}$, as well as $6 / 6$ visual acuity in the right eye according to the Snellen chart.

Fundoscopy revealed a pale optic disc in the left eye and normal retinas (Fig. 1). There was re-duced color vision in the left eye (2/12 of Ishihara plates), and a left relative afferent papillary defect was present. Visual field mapping revealed complete left visual field loss and a normal right-sided visual field (Fig. 2). The remainder of the neuroophthalmological examination was unremarkable. There was nothing in the patient's history or physical examina-
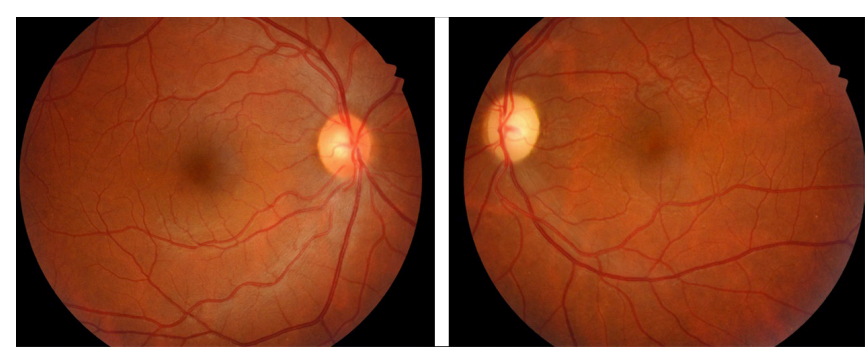

Figure 1. A fundal examination revealed a pale left optic disc.

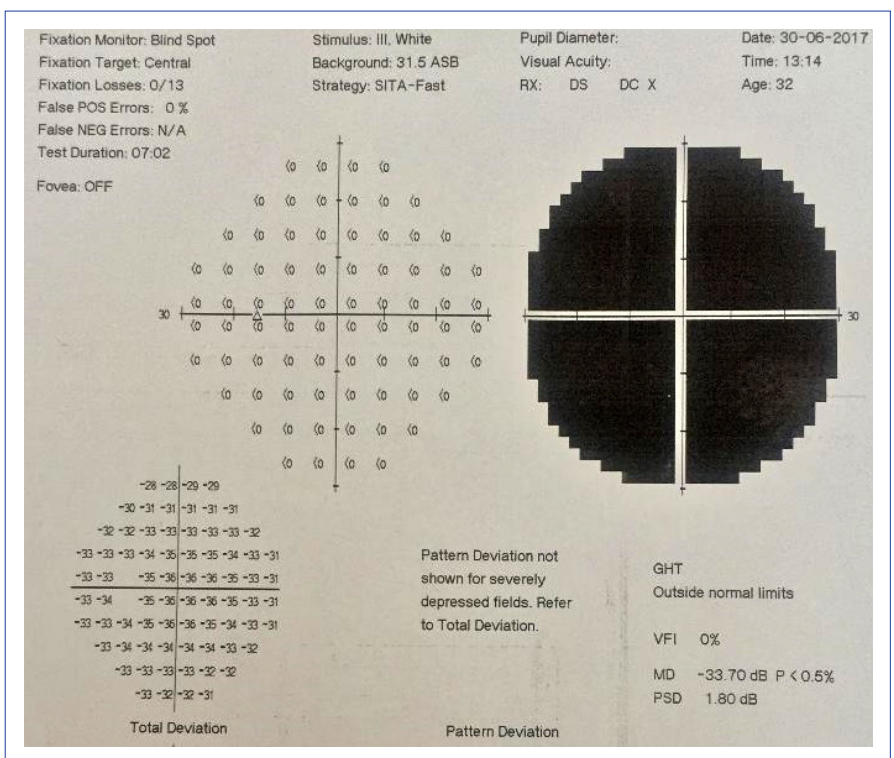

Figure 2. Visual field mapping showed complete visual field loss on the left. tion that was sug-gestive of connective tissue disease or sarcoidosis. Initial blood tests showed a normal full blood count, normal levels of urea and electrolytes, normal liver function tests, and normal C-reactive protein (CRP: $<1$ $\mathrm{mg} / \mathrm{L}$ ), plasma viscosity, and glycated hemoglobin values. A chest X-ray was also normal. A lumbar puncture was performed, which yielded cerebrospinal fluid with normal white and red blood cell counts, a normal angiotensionconverting enzyme (ACE) level, and no oligoclonal bands. A magnetic resonance imaging (MRI) scan of the patient's brain and spinal cord was normal. The Mantoux test and a collagen workup (antinuclear antibody, an-tiphospholipid antibody, and rheumatoid factor) were negative. The serum ACE level was nor-mal. Hepatitis B antigen and HIV serology were non-reactive. $A$ test for serum immunoglobulin $\mathrm{G}(\mathrm{lgG})$ neuromyelitis optica (NMO) antibody was negative. A visually evoked response test revealed prolonged P100 latency in both eyes. Optical coherence tomography (OCT) demon-strated reduced retinal nerve fiber layer (RNFL) thickness (Fig. 3). Due to acute and painful vision loss, a diagnosis of unilateral acute retrobulbar ON was made. Visual acuity improved to $6 / 10$ (Snellen chart) following administration of intravenous steroids (methylprednisolone, $1 \mathrm{~g}$ ) for 3 days and oral methylprednisolone at $1 \mathrm{mg} / \mathrm{kg}$ for the following 11 days.

\section{Discussion}

ON is frequently limited to a single episode. However, some $3 \%$ to $5 \%$ of patients experience recurrent episodes (affecting either eye or both eyes sequentially or simultaneously), along with a negative workup for MS, NMO, and other causes..$^{[2]}$ A decade ago, 2 observations were made almost simultaneously: the description of CRION and the discovery of a specific auto-antibody (NMO-lgG) for NMO. ${ }^{[3,5]}$ These were important for patient management targeted at the preservation of vision because in contrast to MS, there

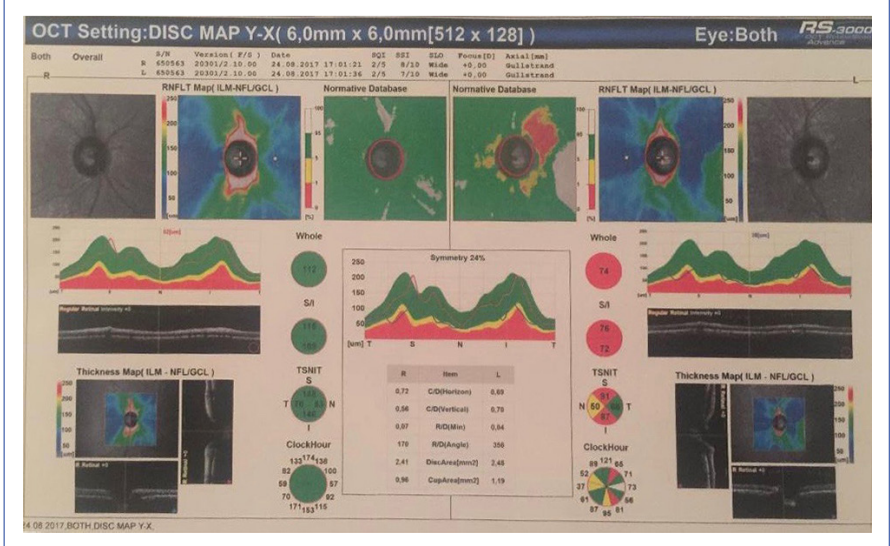

Figure 3. Optical coherence tomography demonstrated reduced retinal nerve fiber layer thickness. 
is a considerable risk of blindness with CRION. ${ }^{[4]}$ Immunosuppressive treatment may allow for the discontinuation of steroid use and prevent or reduce further relapses in cases of CRION, which is dissimilar from MS in its clinical characteristics and natural history. ${ }^{[4]}$

In view of these recurrent steroid-responsive attacks of ON, a differential diagnosis for other demyelinating disorders, such as MS or an NMO spectrum disorder, as well as other causes of secondary demyelination, must be thoroughly considered. In our case, all the ancillary investiga-tions, patient history, and physical examination indications were negative for these etiologies. In addition, signs related to ON were unremarkable upon neurological examination. A gadolinium-enhanced cranial MRI revealed normal findings elsewhere in the CNS, even on follow-up stud-ies, and a test for a serum IgG-NMO antibody was negative.

Recently, Petzold et al. ${ }^{[6]}$ proposed the following 5 diagnostic criteria after a systematic review of 122 reported cases: ON and at least 1 relapse, objective evidence of loss of visual function, seronegative for NMO-lgG, contrast enhancement of acutely inflamed optic nerves on MRI, and response to immunosuppressive treatment and relapse on withdrawal or dose reduction of immunosuppressive treatment. In contrast with the original report of Kidd et al., ${ }^{[3]}$ bilateral (se-quential) loss of vision and pain were not included as criteria in this study. ${ }^{[6]}$ The present case conformed to these new criteria.

The strong response to immuno-suppressive treatment suggests that the etiology of CRION is at least partially immune-mediated. Colpak et al. ${ }^{[7]}$ reported that brain white matter (WM) that appeared normal on a brain MRI demonstrated widespread abnormalities in a cohort of CRION patients when assessed via diffusion tensor imaging (DTI). DTI is an advanced MRI technique that allows professionals to evaluate tissue integrity, primarily WM. Colpak et al. ${ }^{[7]} \mathrm{con}-$ cluded that not only the optic chiasm but also additional WM structures in the brain were affected in patients with CRION. Moreover, their findings may reflect a more complex combination of pathological processes, rather than merely demyelination or axonal degeneration, occurring in the cerebral WM in this particular disease. Their findings thus suggest that CRION is distinct from primary demyelinating diseases, such as MS and NMO.

OCT is an additional diagnostic tool and can also be used to monitor disease progression. RNFL thickness decreases in MS patients, especially those with a history of ON. ${ }^{[8]}$ Bichuetti et al. ${ }^{[9]}$ found that RNFL thickness was significantly worse in NMO and CRION eyes compared with MS patients with a history of recurrent relapsing ON, but they also found no significant differences between NMO and
CRION eyes and concluded that RNFL values can help in differentiating optic neuritis in MS from NMO and CRION. We found significantly reduced RNLF thickness in our patient.

To date, there have been no systematic studies evaluating the duration and intensity of immuno-suppression required in patients with CRION. The data are purely observational, being based on personal experience. Our patient's results indicate that long-term, low-dose corticosteroids are a safe and effective treatment option. Of course, as in all patients who require long-term steroids, osteoporosis prophylaxis should be considered on the basis of national or local guide-lines. Alternatively, as a long-term treatment, intravenous immunoglobulin has been found to be an effective steroid-sparing agent in selected cases. ${ }^{[10]}$ The addition of a steroid-sparing medi-cation, such as azathioprine, methotrexate, cyclophosphamide, mycophenolate, or cyclosporine, has also been reported as a long-term treatment option. ${ }^{[6]}$

\section{Conclusion}

In conclusion, identifying these patients has therapeutic implications. CRION is highly respon-sive to steroids, but there is a considerable risk of blindness if these steroids are not adminis-tered. CRION should be diagnosed after excluding the long list of diseases reviewed here, es-pecially MS and NMO. Although, at present, there are no systemic studies evaluating the dura-tion and intensity of immunosuppression required in patients with CRION, our patient's results indicate that administration of an intravenous steroid for 3 days and oral methylprednisolone $1 \mathrm{mg} / \mathrm{kg}$ for the following 11 days represents a safe and effective treatment option.

\section{Disclosures}

Informed Consent: Written informed consent was obtained from the patient for the publication of the case report and the accompanying images.

Peer-review: Externally peer-reviewed.

Conflict of Interest: None declared.

Authorship Contributions: Concept - E.T.Y., B.B.; Design - E.T.Y., B.B.; Supervision - E.T.Y., B.B.; Materials -E.T.Y., B.B.; Data collection \&/or processing - E.T.Y., B.B.; Analysis and/or interpretation - E.T.Y., B.B.; Literature search -E.T.Y., B.B.; Writing - E.T.Y., B.B.; Critical review - E.T.Y., B.B.

\section{References}

1. Rodriguez M, Siva A, Cross SA, O'Brien PC, Kurland LT. Optic neuritis: a population-based study in Olmsted County, Minnesota. Neurology 1995;45:244-50.

2. Pirko I, Blauwet LA, Lesnick TG, Weinshenker BG. The natural history of recurrent optic neuritis. Arch Neurol 2004;61:1401-5. 
3. Kidd D, Burton B, Plant GT, Graham EM. Chronic relapsing inflammatory optic neuropathy (CRION). Brain 2003;126:276-84.

4. de Seze J. Inflammatory Optic Neuritis: From Multiple Sclerosis to Neuromyelitis Optica. Neuroophthalmology 2013;37:141-5.

5. Lennon VA, Wingerchuk DM, Kryzer TJ, Pittock SJ, Lucchinetti CF, Fujihara $\mathrm{K}$, et al. A serum autoantibody marker of neuromyelitis optica: distinction from multiple sclerosis. Lancet 2004;364:210612.

6. Petzold A, Plant GT. Chronic relapsing inflammatory optic neuropathy: a systematic review of 122 cases reported. J Neurol 2014;261:17-26.

7. Colpak Al, Kurne AT, Oguz KK, Has AC, Dolgun A, Kansu T. White matter involvement beyond the optic nerves in CRION as as- sessed by diffusion tensor imaging. Int J Neurosci 2015;125:10-7.

8. Daldal H, Tok OY, Sengeze N, Koyuncuoglu HR, Tok L, Ozkaya D, et al. Retinal Nerve Fiber Layer Thickness in Multiple Sclerosis. Turk J Ophthalmol 2011;41:1-5.

9. Bichuetti DB, de Camargo AS, Falcão AB, Gonçalves FF, Tavares $I M$, de Oliveira EM. The retinal nerve fiber layer of patients with neuromyelitis optica and chronic relapsing optic neuritis is more severely damaged than patients with multiple sclerosis. J Neuroophthalmol 2013;33:220-4.

10. Stiebel-Kalish H, Hammel N, van Everdingen J, Huna-Baron R, Lee AG. Intravenous immunoglobulin in recurrent-relapsing inflammatory optic neuropathy. Can J Ophthalmol 2010;45:71-5. 